\title{
Vascular abnormalities and development of hypoxia in microscopic melanoma xenografts
}

\author{
Jon-Vidar Gaustad* (D), Trude G. Simonsen, Lise Mari K. Andersen and Einar K. Rofstad
}

\begin{abstract}
Background: Studies investigating the oxygenation status and the development of hypoxia in microscopic tumors are sparse. The purpose of this study was to measure the extent of hypoxia in microscopic melanoma xenografts and to search for possible mechanisms leading to the development of hypoxia in these tumors.

Methods: A-07, D-12, R-18, and U-25 human melanoma xenografts grown in dorsal window chambers or as flank tumors were used as preclinical tumor models. Morphologic and functional parameters of vascular networks were assessed with intravital microscopy, and the expression of angiogenesis-related genes was assessed with quantitative PCR. Microvessels, pericytes, and the extent of hypoxia were assessed by immunohistochemistry in microscopic tumors by using CD31, aSMA, and pimonidazole as markers, and the extent of radiobiological hypoxia was assessed in macroscopic flank tumors.
\end{abstract}

Results: Macroscopic R-18 and U-25 tumors showed extensive hypoxia, whereas macroscopic A-07 and D-12 tumors were less hypoxic. R-18 and U-25 tumors developed hypoxic regions before they reached a size of 2-3 mm in diameter, whereas A-07 and D-12 tumors of similar size did not show hypoxic regions. The development of hypoxic regions was not caused by low vessel density, but was rather a result of inadequate vascular function. Inadequate vascular function was not caused by low vessel diameters or long vessel segments, but was associated with poor vascular pericyte coverage. Poor pericyte coverage was associated with the expression of eight angiogenesis-related genes.

Conclusions: Two of the four investigated melanoma models developed hypoxic regions in microscopic tumors, and the development of hypoxia was associated with poor vascular pericyte coverage and inadequate vascular function.

Keywords: Microscopic tumors, Malignant melanoma, Vessel density, Vascular function, Vascular pericyte coverage, Tumor hypoxia, Angiogenic profiles

\section{Background}

Most tumors are heterogeneous in oxygen tension and develop regions with hypoxic cells during growth $[1,2]$. Preclinical studies have demonstrated that tumors with extensive hypoxic regions are resistant to several types of therapy including radiation therapy, immunotherapy, and some forms of chemotherapy, and that tumor hypoxia may promote malignant progression and metastatic dissemination [1-4]. Clinical studies have shown that patients with highly hypoxic tumors have increased frequency of locoregional treatment failure, increased

\footnotetext{
${ }^{*}$ Correspondence: Jon.Vidar.Gaustad@rr-research.no

Group of Radiation Biology and Tumor Physiology, Department

of Radiation Biology, Institute for Cancer Research, Oslo University

Hospital, Montebello, 0310 Oslo, Norway
}

incidence of distant metastases and poor disease-free and overall survival rates following radiation therapy alone or radiation therapy combined with surgery and/or chemotherapy [1].

Tumor hypoxia is a consequence of inadequate oxygen supply caused by extensive abnormalities in the vascular network [2]. The vascular abnormalities include low and heterogeneous vessel density, elongated and tortuous vessels, aberrant vessel diameters, and vessel wall abnormalities (i.e., incomplete endothelial lining, interrupted basement membrane, and lack of pericytes and contractile vessel wall components) $[2,5,6]$. These morphological abnormalities collectively increase the geometric resistance to blood flow and impair vascular function [7]. Studies of macroscopic experimental tumors have shown 
that the severity of the vascular abnormalities increases during tumor growth, resulting in reduced blood supply and increased extent of hypoxia [8].

Studies investigating the development of hypoxia in microscopic tumors are sparse, but the extent of hypoxia has been measured in microscopic HT29 and HCT-8 colorectal adenocarcinoma xenografts [9]. In these models, tumors with diameters less than $1 \mathrm{~mm}$ were avascular and highly hypoxic, whereas tumors with diameters of 1-4 mm were vascularized and did not show hypoxic regions. The study suggested that clinical micrometastases may be resistant to ionizing radiation and chemotherapy before they induce angiogenesis, and that vascularized micrometastases may not show hypoxiainduced treatment resistance.

In the current study, we investigated the extent of hypoxia in microscopic A-07, D-12, R-18, and U-25 melanoma xenografts growing in dorsal window chambers. To search for mechanisms for the development of hypoxia, the morphology and function of the tumor vascular networks were studied with intravital microscopy and immunohistochemistry, and the expression of several angiogenesis-related genes was measured with quantitative PCR. The hypoxic status of microscopic tumors was compared with the extent of hypoxia in macroscopic tumors of the same melanoma models.

\section{Methods}

\section{Tumor models}

A-07, D-12, R-18, and U-25 human melanoma cells [10] were constitutively transfected with green fluorescence protein by lipofection, and the transfected cells used in this study were obtained from our frozen stock. Window chambers were surgically implanted in the dorsal skin fold of adult female BALB/c $n u / n u$ mice as reported earlier [11]. Tumors were initiated by implanting multicellular spheroids or solid tumor pieces in window chambers, or by inoculating approximately $3.5 \times 10^{5}$ cells intradermally in the mouse flank. Female mice were used for practical reasons.

\section{Anesthesia}

Window chamber implantation and intravital microscopy examinations were carried out with anesthetized mice. Fentanyl citrate (Janssen Pharmaceutica, Beerse, Belgium), fluanisone (Janssen Pharmaceutica), and midazolam (Hoffmann-La Roche, Basel, Switzerland) were administered intraperitoneally in doses of $0.63,20$, and $10 \mathrm{mg} / \mathrm{kg}$, respectively.

\section{Intravital microscopy}

Mice with window chambers were fixed to the microscope stage during intravital microscopy, and the body core temperature was kept at $37-38{ }^{\circ} \mathrm{C}$ by using a hot-air generator. Imaging was performed by using an inverted fluorescence microscope (IX-71; Olympus, Munich, Germany) and a black and white CCD camera (C9300024; Hamamatsu Photonics, Hamamatsu, Japan). Tumor vasculature was visualized by using a $\times 4$ objective lens, transillumination, and filters for green light, resulting in images with a pixel size of $3.7 \times 3.7 \mu \mathrm{m}^{2}$. Vessel density (i.e., total vessel length per $\mathrm{mm}^{2}$ tumor area), median intercapillary distance (i.e., the distance from a tumor vessel to the nearest neighboring vessel), and median vessel diameter were computed from manually produced vascular masks of the entire vascular networks, as previously described [11]. Median vessel segment length was calculated from $\sim 50$ randomly selected vessel segments. To investigate vascular function, first-pass imaging movies were recorded after a $0.2 \mathrm{~mL}$ bolus of $50 \mathrm{mg} / \mathrm{mL}$ tetramethylrhodamine isothiocyanate-labeled dextran (Sigma-Aldrich, Schnelldorf, Germany) with a molecular weight of $155 \mathrm{kDa}$ was injected into the lateral tail vein. First-pass imaging movies were recorded at a rate of 22.3 frames per second by using a $2 \times$ objective lens, resulting in a time resolution of $44.8 \mathrm{~ms}$ and a pixel size of $7.5 \times 7.5 \mu \mathrm{m}^{2}$. Blood supply time (BST) images were produced by assigning a BST value to each pixel of the vascular masks [11]. The BST of a pixel was defined as the time difference between the frame showing maximum fluorescence intensity in the pixel and the frame showing maximum fluorescence intensity in the main tumor supplying artery, as described in detail previously [12].

\section{Immunohistochemical detection of tumor hypoxia, microvessels, and pericytes}

The tumors were resected immediately after the intravital microscopy examinations and fixed in phosphate-buffered $4 \%$ paraformaldehyde. Pimonidazole [1-[(2-hydroxy3-piperidinyl)-propyl]-2-nitroimidazole], administered as described previously [13], was used as a hypoxia marker, CD31 was used as a marker for endothelial cells, and $\alpha$-smooth muscle actin ( $\alpha \mathrm{SMA})$ was used as a marker for pericytes. Immunohistochemistry was done by using a peroxidase-based indirect staining method [13]. An antipimonidazole rabbit polyclonal antibody (gift from Prof. J.A. Raleigh, Department of Radiation Oncology, University of North Carolina School of Medicine, Chapel Hill, $\mathrm{NC}$ ), an anti-CD31 rabbit polyclonal antibody (Ab28364; Abcam, Cambridge, United Kingdom), or an anti- $\alpha$ SMA rabbit polyclonal antibody (Ab5694; Abcam) was used as primary antibody. Diaminobenzidine was used as chromogen, and hematoxylin was used for counterstaining. Hypoxic area fractions were determined by image analysis. Number of CD31-positive and $\alpha$ SMA-positive profiles per $\mathrm{mm}^{2}$ of tumor tissue $\left(\# / \mathrm{mm}^{2}\right)$ was scored manually. 


\section{Radiobiological assessment of tumor hypoxia}

The paired survival curve method was used to measure radiobiological hypoxia [13]. Tumors were irradiated under air-breathing or hypoxic conditions at a dose rate of $5.1 \mathrm{~Gy} / \mathrm{min}$ by using an X-ray unit operated at $220 \mathrm{kV}$, 19-20 mA, and with $0.5 \mathrm{~mm} \mathrm{Cu}$ filtration. Hypoxic conditions were obtained by occluding the tumor blood supply with a clamp $5 \mathrm{~min}$ before irradiation. Tumor cell survival was measured in vitro. The tumors were resected immediately after irradiation, minced in cold Hanks' balanced salt solution, and treated with an enzyme solution ( $0.2 \%$ collagenase, $0.05 \%$ pronase, and $0.02 \%$ DNase) at $37{ }^{\circ} \mathrm{C}$ for $2 \mathrm{~h}$. Trypan blue-negative cells were plated in $25 \mathrm{~cm}^{2}$ tissue culture flasks and incubated at $37{ }^{\circ} \mathrm{C}$ for 14 days for colony formation. The cell surviving fraction of an irradiated tumor was calculated from the number of cells plated, the number of colonies counted, and the mean plating efficiency of six untreated control tumors. Cell survival curves were established for clamped and unclamped tumors, and the fraction of radiobiologically hypoxic cells $\left(\mathrm{HF}_{\mathrm{RAD}}\right)$ was calculated from the vertical displacement of the curves [13].

\section{Quantitative PCR}

RNA isolation, cDNA synthesis, and quantitative PCR were performed as described in detail previously [14]. Briefly, gene expression was assessed by using the $\mathrm{RT}^{2}$ Profiler PCR Array Human Angiogenesis (PAHS-024A) from SABiosciences (Frederick, MD). Real-time PCR was performed on an ABI 7900HT Fast Real-Time PCR instrument (Applied Biosystems, Carlsbad, CA). Each tumor line was run in three biological replicates. Glyceraldehyde-3-phosphate dehydrogenase (GAPDH) and $\beta$-actin (ACTB) were used as normalization genes because these housekeeping genes showed stable expression across the melanoma lines studied here. Thus, each replicate $C_{T}$ value was normalized to the mean $C_{T}$ value of GAPDH and ACTB $\left(\Delta C_{T}=C_{T}\right.$ gene of interest $-C_{T}$ mean of GADPH and ACTB $)$. The normalized expression level of each gene was calculated from the three biological replicates as $2^{- \text {mean } \Delta \mathrm{CT}}$.

\section{Statistical analysis}

Statistical comparisons of data were performed by one-way analysis of variance followed by the StudentNeuman-Keuls test when the data complied with the conditions of normality and equal variance, and under other conditions by the Kruskal-Wallis one-way analysis of variance on ranks followed by the Dunn's test. Probability values of $P<0.05$, determined from two-sided tests, were considered significant. The statistical analysis was performed by using the SigmaStat statistical software (SPSS Science, Chicago, IL).

\section{Results}

$\mathrm{HF}_{\mathrm{RAD}}$ was measured in macroscopic flank tumors $\left(\sim 400 \mathrm{~mm}^{3}\right)$ by using the paired survival curve method. Figure 1a shows paired survival curves for A-07, D-12, $\mathrm{R}-18$, and U-25 melanoma xenografts. $\mathrm{HF}_{\mathrm{RAD}}$ was significantly higher in R-18 and U-25 tumors than in A-07 and D-12 tumors, and significantly higher in D-12 tumors than in A-07 tumors (Fig. $1 \mathrm{~b} ; P<0.01$ ).

To investigate the development of hypoxia in microscopic tumors, A-07, D-12, R-18, and U-25 tumors were initiated in dorsal window chambers and subjected to histological examination. Figure 2a shows immunohistochemical preparations of representative A-07, D-12, R-18, and U-25 tumors stained for pimonidazole. $78 \%$ of the R-18 tumors and $83 \%$ of the U-25 tumors had developed hypoxic regions before they reached a size of 2-3 mm in diameter, whereas A-07 and D-12 tumors with similar sizes did not show hypoxic regions (Fig. 2). The fraction of pimonidazole-positive cells $\left(\mathrm{HF}_{\mathrm{PIM}}\right)$ was thus significantly higher in R-18 and U-25 tumors than in A-07 and D-12 tumors (Fig. 2b; $P<0.01$ ).

The microscopic tumors were subjected to intravital microscopy multiple times during growth. Figure 3a shows intravital microscopy images of a representative A-07 tumor, and illustrates the first signs of angiogenesis (small bleedings within the tumor mass; day 3), novel tumor vessels (day 5), and an established vascular network (day 7 and day 10). In D-12, R-18, and U-25 tumors, the first tumor vessels appeared later, and the tumor growth rates were significantly lower than in A-07 tumors (Fig. 3b; $P<0.05$ ). U-25 tumors showed lower growth rates than D-12 and R-18 tumors $(P<0.05)$, whereas the hypoxia-positive R-18 tumors did not differ from the hypoxia-negative D-12 tumors in growth rate $(P>0.05)$.

The melanoma models were allowed different growth times before they were resected for histological examination in accordance with the differences in tumor growth rate (Fig. 3b). Figure 4a shows high resolution intravital microscopy images of the vasculature of representative A-07, D-12, R-18, and U-25 tumors immediately before the tumors were resected. At this time point, all tumors were highly vascularized. The vessel segments were uniformly distributed, and avascular regions were not observed in any of the tumors. A-07 tumors showed higher vessel density and lower intercapillary distance than D-12, R-18, and U-25 tumors (Fig. 4b, c; $P<0.05$ ). A-07 tumors also showed larger vessel diameters than D-12, R-18, and U-25 tumors (Fig. 4d; $P<0.05$ ), whereas R-18 tumors showed longer vessel segments than A-07 and U-25 tumors (Fig. 4e; $P<0.05$ ). None of these morphological parameters distinguished between the hypoxia-positive (R-18 and U-25) and the hypoxia-negative tumors (A-07 and D-12). 

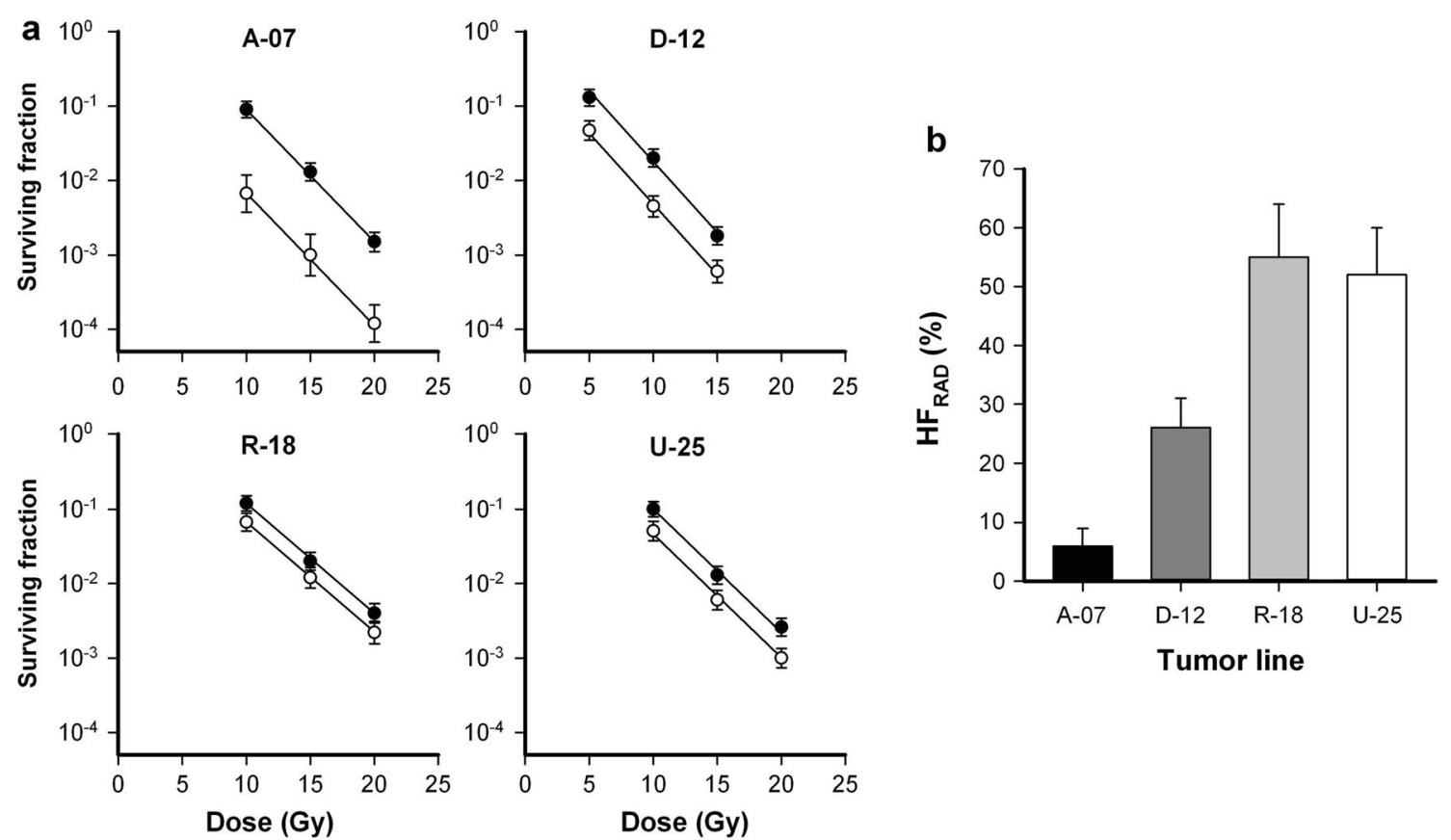

Fig. 1 Hypoxia in macroscopic tumors. a Cell survival curves of $\sim 400 \mathrm{~mm}^{3}$ flank tumors irradiated under air breathing (open circle) or hypoxic (filled circle) conditions. Points, geometric mean of 5-7 tumors; bars, SE. b Fraction of radiobiologically hypoxic cells (HF $F_{\text {RAD }}$ ) in A-07, D-12, R-18, and U-25 tumors. $\mathrm{HF}_{\text {RAD }}$ was calculated from the survival curves in a. Columns, mean values; bars, SE
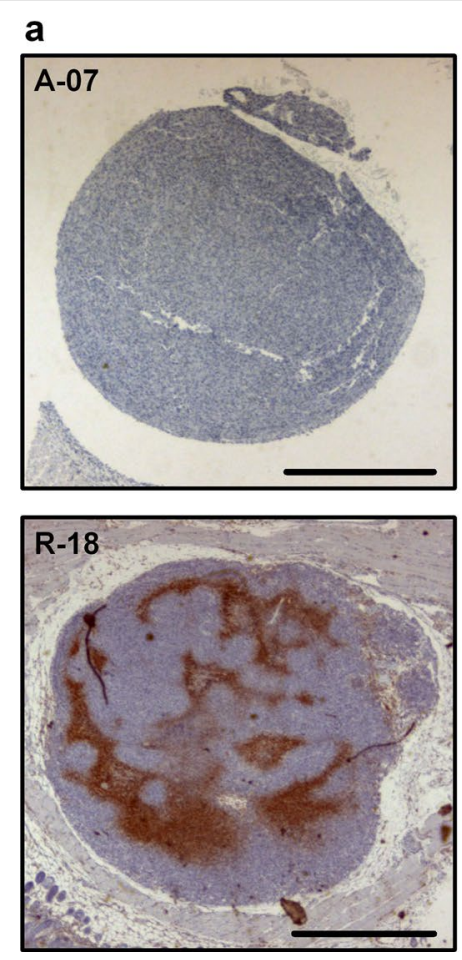

Fig. 2 Hypoxia in microscopic tumors. a Immunohistochemical preparations of representative microscopic A-07, D-12, R-18, and U-25 window

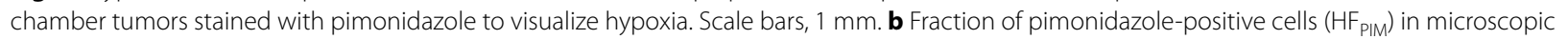
A-07, D-12, R-18, and U-25 window chamber tumors. Points, individual tumors; horizontal lines, means 

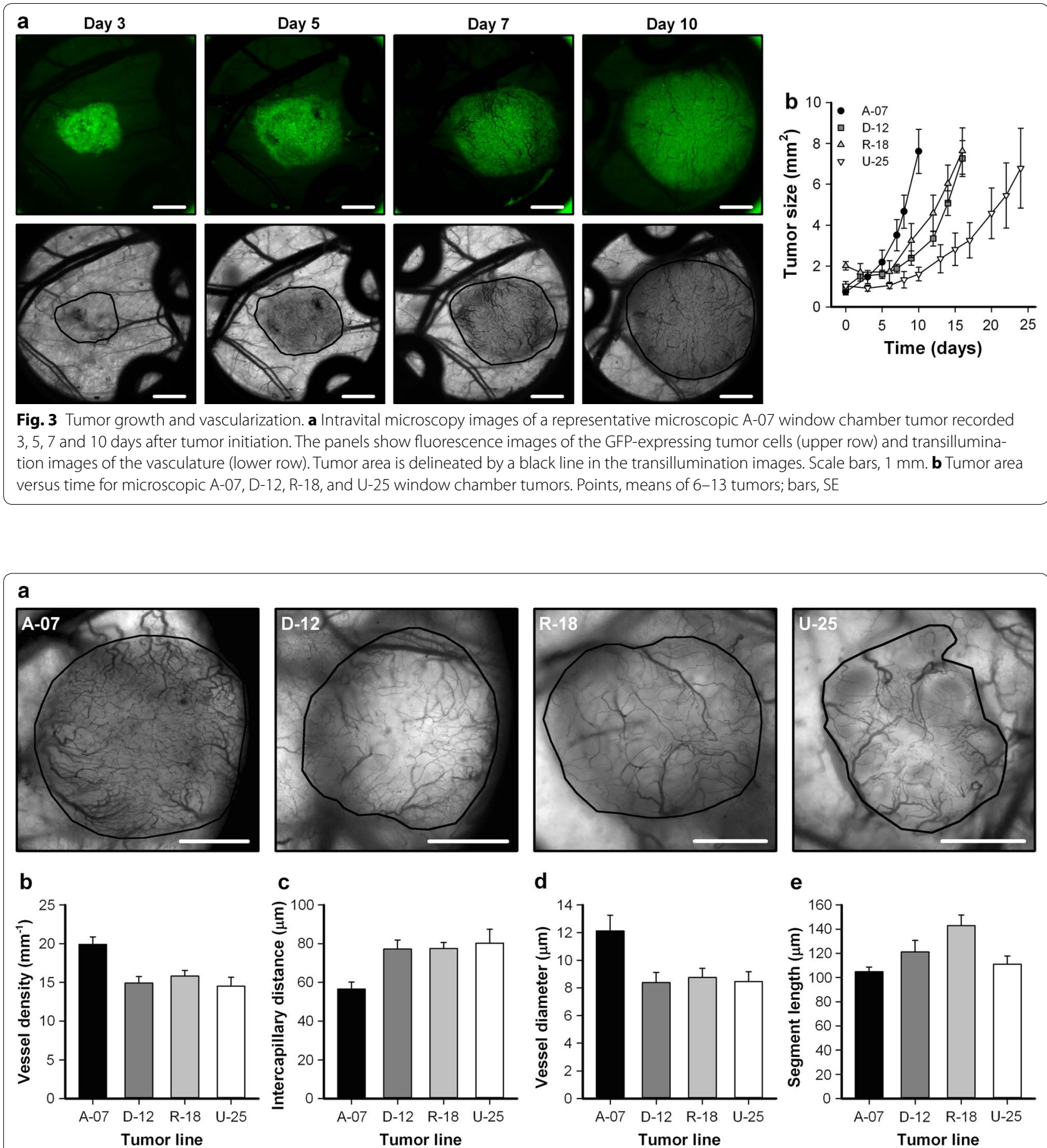

Fig. 4 Vascular morphology. a Intravital microscopy images of representative microscopic A-07, D-12, R-18, and U-25 window chamber tumors. Tumor area is delineated by a black line. Scale bars, $1 \mathrm{~mm}$. b-e Vessel density $(\mathbf{b})$, median intercapillary distance (c), median vessel diameter (d), and median vessel segment length (e) in microscopic A-07, D-12, R-18, and U-25 window chamber tumors. Columns, means of 6-13 tumors; bars, SE

Adjacent histological tumor sections were stained with CD31 to visualize microvessels or with $\alpha$ SMA to visualize pericytes. CD31 positive vessels with or without positive $\alpha$ SMA staining are highlighted in Fig. 5a with white or black arrows, respectively. In A-07 and D-12 tumors, most CD31 positive vessels showed positive $\alpha$ SMA staining, and long bands with positive $\alpha$ SMA staining were observed between microvessels. In contrast, only a few 
CD31 positive vessels showed positive $\alpha$ SMA staining in R- 18 and U-25 tumors, and positive $\alpha$ SMA staining were only found adjacent to microvessels. In agreement with the intravital microscopy observations, the density of CD31 positive vessels was higher in A-07 tumors than in D-12, R-18, and U-25 tumors (Fig. 5b; $P<0.05$ ). The density of $\alpha$ SMA positive profiles was higher in A-07 tumors than in D-12, R-18, and U-25 tumors, and higher in D-12 tumors than in R-18 and U-25 tumors (Fig. 5c; $P<0.05$ ). Moreover, the ratio between $\alpha \mathrm{SMA}$ and CD31 positive profiles was higher for A-07 and D-12 tumors than for R-18 and U-25 tumors (Fig. 5d; $P<0.05$ ), and did not differ between A-07 and D-12 tumors (Fig. 5d; $P>0.05$ ).
To investigate the function of the vascular networks, first-pass imaging movies were recorded and BST images and BST frequency distributions were produced. The BST image and the BST frequency distribution of representative A-07, D-12, R-18, and U-25 tumors are shown in Fig. 6a. D-12 tumors showed lower BST values than R-18 and U-25 tumors (Fig. 6b; D-12 vs R-18: $P<0.01$; D-12 vs U-25: $P=0.012$ ), and A-07 tumors showed lower BST values than $\mathrm{R}-18$ tumors (Fig. $6 \mathrm{~b} ; P<0.01$ ).

The expression of 84 known angiogenesis-related genes was quantified by using a commercial quantitative PCR array. The genes encoding basic fibroblast growth factor (bFGF), epiregulin (EREG), basic helix-loop-helix
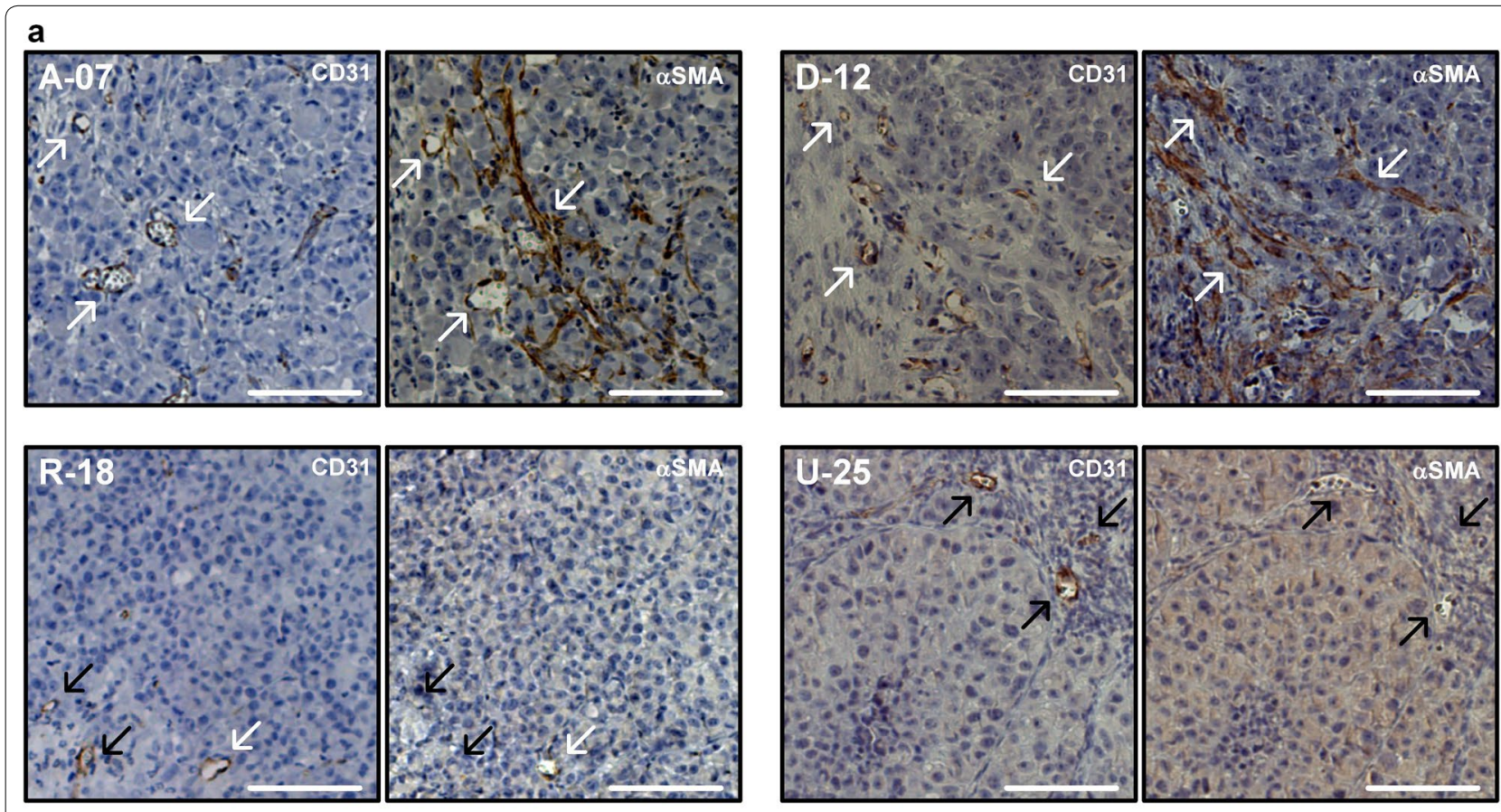

b

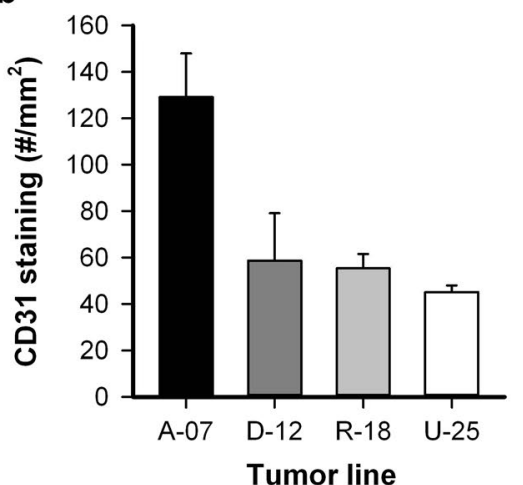

C

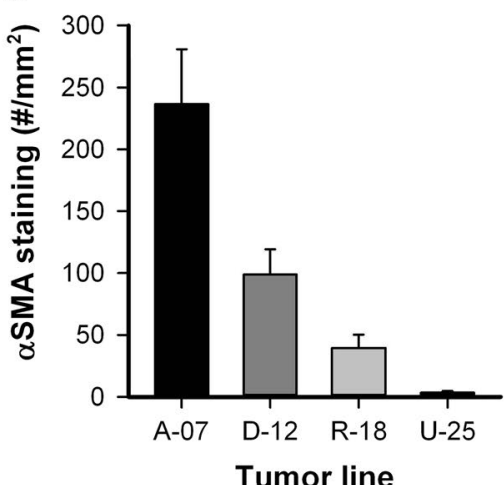

d

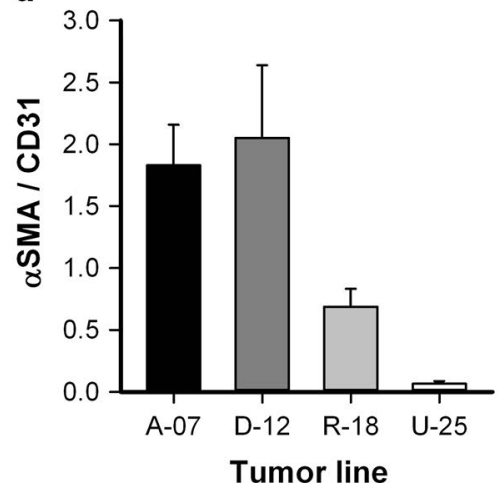

Fig. 5 Immunohistological assessment of microvessels and pericytes. a Adjacent immunohistological sections of representative microscopic A-07, D-12, R-18, and U-25 window chamber tumors stained with CD31 to visualize microvessels or aSMA to visualize pericytes. Examples of CD31 positive vessels with or without aSMA staining are highlighted with white or black arrows, respectively. Scale bars, $100 \mu \mathrm{m}$. b-d Density of CD31 positive profiles $(\mathbf{b})$, density of aSMA positive profiles $(\mathbf{c})$, and the ratio between aSMA and CD31 positive profiles (d) 
transcription factor (HAND2), neuropilin 1 (NRP1), urokinase-type plasminogen activator (uPA), and vascular endothelial growth factor C (VEGFC) showed higher expression (> fivefold) in the A-07 and D-12 models than in the R-18 and U-25 models (Fig. 7a), whereas the genes encoding transforming growth factor $\beta$ receptor 1 (TGFBR1) and VEGFD showed higher expression (> fivefold) in the R-18 and U-25 models than in the A-07 and D-12 models (Fig. 7b).

\section{Discussion}

Tumor hypoxia was assessed by using a radiobiological assay in macroscopic tumors. This assay measures the fraction of the clonogenic cells in tumors that are hypoxic [13]. $\mathrm{HF}_{\mathrm{RAD}}$ is considered to be of great clinical relevance, because only the clonogenic cells are relevant for tumor growth and response to treatment [15]. Unfortunately, $\mathrm{HF}_{\mathrm{RAD}}$ cannot be measured without severe problems in microscopic tumors, and consequently
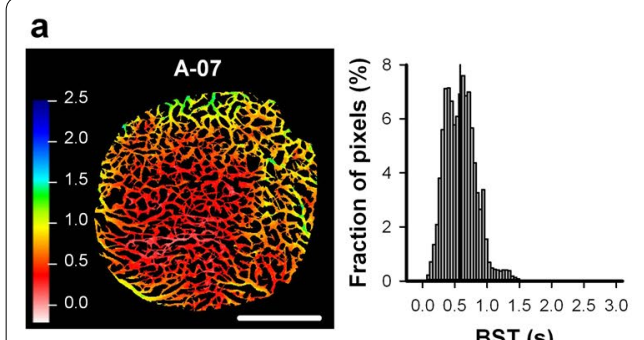

$\operatorname{BST}(\mathbf{s})$
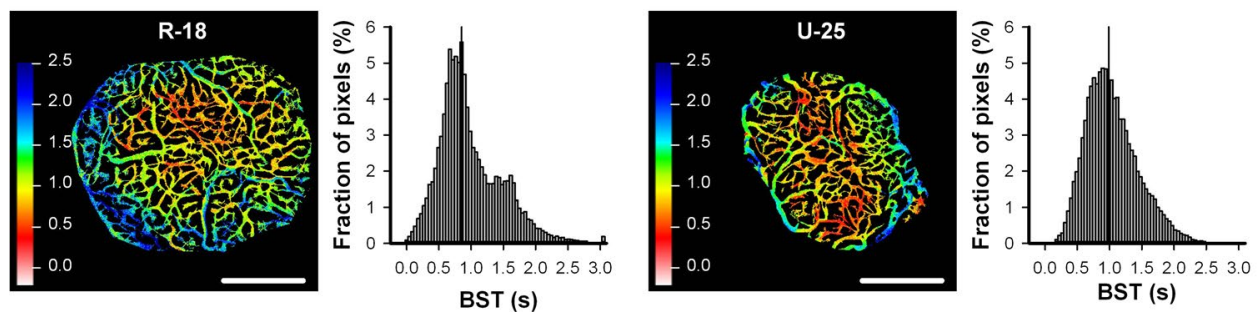

Fig. 6 Vascular function. a Blood supply time (BST) images and the corresponding BST frequency distributions of representative microscopic A-07, D-12, R-18, and U-25 window chamber tumors. Color bars, BST scales in seconds; scale bars, 1 mm. b Median BST in microscopic A-07, D-12, R-18, and U-25 window chamber tumors. Columns, means of 7-17 tumors; bars, SE
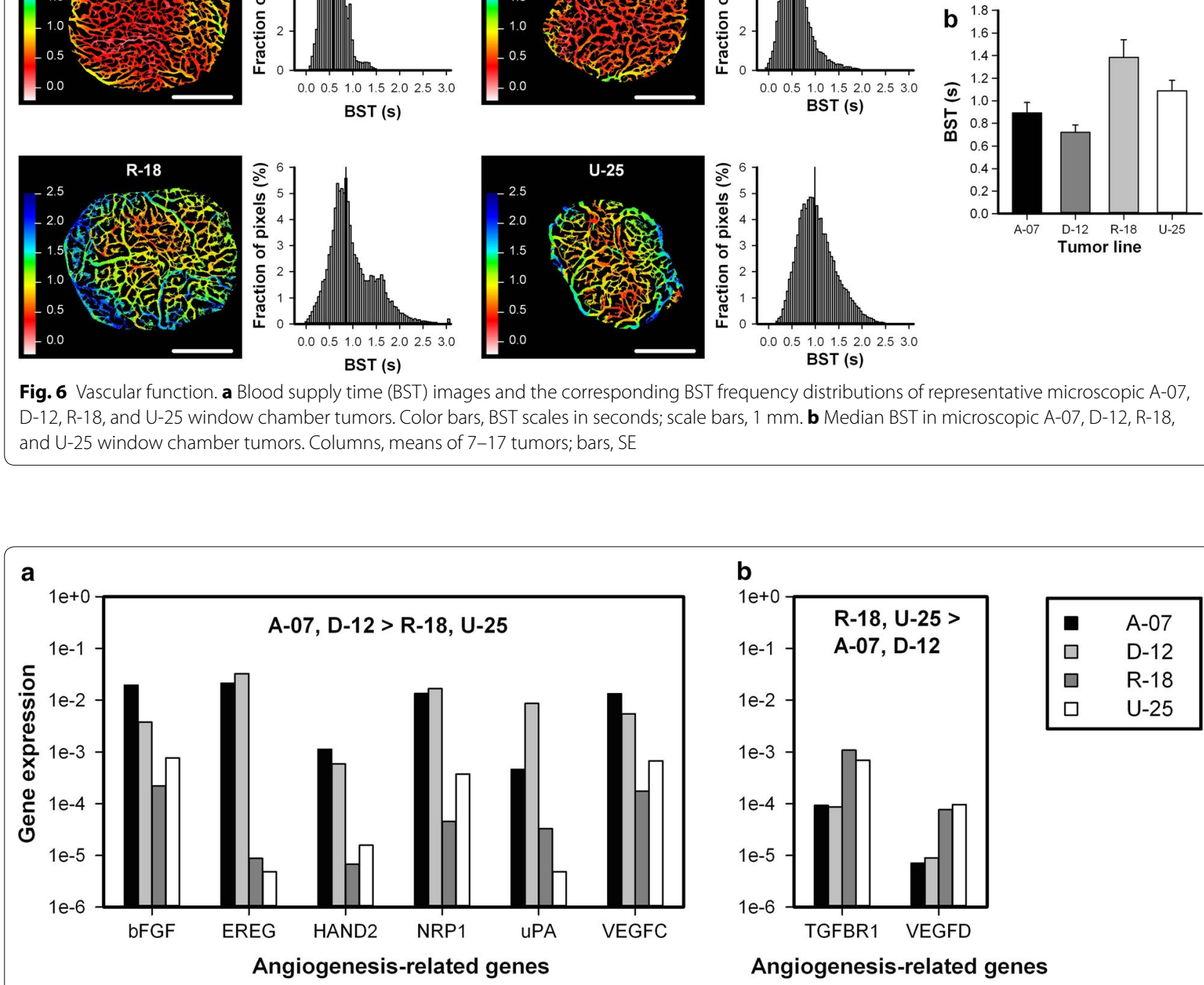

Fig. 7 Expression of angiogenesis-related genes. Normalized expression $\left(2^{-\Delta C T}\right)$ of angiogenesis-related genes that are more than fivefold higher expressed in the A-07 and D-12 models than in the R-18 and U-25 models (a), and more than fivefold higher expressed in the R-18 and U-25 models than in the A-07 and D-12 models (b). Gene expression was measured with quantitative PCR and normalized to housekeeping genes with stable expression (GAPDH and ACTB) 
an immunohistochemical assay using pimonidazole as hypoxia marker was used to detect hypoxia in window chamber tumors. The immunohistochemical assay provides information of the spatial distribution of hypoxia, but does not distinguish between clonogenic and nonclonogenic cells [13]. The absolute values of $\mathrm{HF}_{\mathrm{RAD}}$ were substantially higher than the absolute values of $\mathrm{HF}_{\mathrm{PIM}}$. These differences probably reflected the different cell types that were measured with the assays (clonogenic versus all cells) and were also influenced by the differences in tumor size (macroscopic versus microscopic tumors). In addition, $\mathrm{HF}_{\mathrm{RAD}}$ is sensitive to both chronically and acutely hypoxic cells whereas the immunohistochemical assay has been optimized to preferentially stain chronically hypoxic cells [13]. Despite these differences, the melanoma models that developed hypoxia in microscopic tumors (R-18 and U-25) also showed the highest $\mathrm{HF}_{\mathrm{RAD}}$ in macroscopic tumors. This observation suggests that hypoxia was caused by tumor-line specific abnormalities, but does not imply that the hypoxic status of tumors is static. Thus it is well known that the extent of tumor hypoxia can increase during growth and may be altered by treatments [8]. We have previously shown that subcurative radiation and antiangiogenic treatment can increase the hypoxic fractions in the melanoma models included in the current study $[16,17]$.

R-18 and U-25 tumors developed hypoxic regions before they reached a size of $2-3 \mathrm{~mm}$ in diameter, whereas A-07 and D-12 tumors of similar sizes did not show hypoxic regions. Hypoxia was not caused by low vessel densities in microscopic tumors because all the tumors were highly vascularized, and the hypoxic R-18 and U-25 tumors showed similar vessel densities as the non-hypoxic D-12 tumors. By using typical values for intracapillary oxygen tensions and tumor tissue oxygen consumption rates, the oxygen diffusion distance has been calculated to be approximately $150 \mu \mathrm{m}$ in tumor tissue [2]. These theoretical calculations imply that tumor hypoxia and necrosis can be expected if the intercapillary distance exceeds $300 \mu \mathrm{m}$. The intercapillary distances measured with intravital microscopy were low compared to this critical limit. However, intravital microscopy images show 2-dimensional projections of the 3-dimensional vasculature, and are expected to underestimate intercapillary distances [18]. Intercapillary distances were also calculated from histological sections stained for microvessels by assuming a homogenous vessel distribution. These distances ranged from $\sim 100 \mu \mathrm{m}$ (A-07) to $\sim 150 \mu \mathrm{m}(\mathrm{D}-12, \mathrm{R}-18$, and U-25) and were thus twofold to threefold lower than the critical limit determined by the oxygen diffusion distance [2].

Mathematical simulations and experimental studies have demonstrated that hypoxic regions may develop close to blood vessels if the vascular function is impaired $[2,7,19]$. The hypoxic R-18 and U-25 tumors showed higher BST values and hence lower blood flow velocities than the non-hypoxic A-07 and D-12 tumors, suggesting that development of hypoxia was a result of inadequate vascular function. Narrow and elongated vessels may reduce blood flow due to high geometric resistance [7]. Large vessel diameters may thus have contributed to efficient blood supply in A-07 tumors, and narrow and elongated vessels may have contributed to poor vascular function in R-18 tumors. However, the non-hypoxic D-12 tumors did not differ from hypoxic U-25 tumors in vessel diameter or vessel segment length, suggesting that other parameters were more important in governing vascular function in these tumors.

Pericytes are known to provide structural support to blood vessels and to influence vessel stability [20]. Moreover, depletion of pericytes in genetically engineered mouse models has been shown to impair vascular function and induce hypoxia in breast cancer xenografts [21]. In the present study, $\alpha \mathrm{SMA}$ was used as a marker for pericytes. The majority of the vessels in A- 07 and D-12 tumors showed positive $\alpha$ SMA staining whereas only a few vessels showed positive $\alpha$ SMA staining in R-18 and U-25 tumors. This observation implies that the melanoma models differed in vascular pericyte coverage, and poor pericyte coverage was associated with inadequate vascular function and development of hypoxia. This finding suggests that vascular pericyte coverage may have prognostic value in clinical melanomas, a suggestion that merits clinical investigation. In line with this suggestion, patients with no detectable vascular pericyte coverage had lower disease-free and overall survival rates than patients with detectable pericyte coverage in a study of 130 patients with invasive breast cancer [22]. As with all available pericyte markers, $\alpha \mathrm{SMA}$ is also expressed by some other cell types, including smooth muscle, myofibroblasts, and myoepithelium [20]. It is thus possible that also other cell types were identified by $\alpha$ SMA staining in the present study.

The current study demonstrates that microscopic tumors with high vessel densities may develop regions with hypoxic tissue if the vascular function is inadequate. This finding differs from a study by $\mathrm{Li}$ et al. which reported that vascularized colorectal carcinoma xenografts with a diameter of 1-4 $\mathrm{mm}$ did not show hypoxic regions [9], but is in agreement with a study by Cao et al. which found evidence of hypoxia in vascularized colon carcinoma xenografts with a diameter of 1-2 mm [23]. The finding has important implications for clinical micrometastases because micrometastatic disease is often treated with chemotherapy or regional radiotherapy after the primary tumor has been surgically removed. 
If clinical micrometastases have developed regions with hypoxic tissue, they may be resistant to these treatments [2]. Clinical studies have suggested that the treatment of primary tumors may be improved by combining radiation therapy with hypoxia-modifying agents [24]. It is possible that also the treatment of micrometastatic disease may be improved by using similar strategies to reduce hypoxia-induced treatment resistance.

Several angiogenic stimulators have been shown to be important for initiation and maintenance of angiogenesis in melanoma, including VEGFA, interleukin-8 (IL-8), bFGF, and UPA [25]. In the present study, the A-07 and D-12 models showed higher expression of the genes encoding bFGF, EREG, HAND2, NRP1, uPA, and VEGFC, and lower expression of the genes encoding TGFBR 1 and VEGFD than the R-18 and U-25 models. These genes have been reported to regulate angiogenesis, vascular remodeling, and/or lymphangiogenesis, but their role in regulating vascular function is not clear [25-31]. Moreover, these genes are not found among the genes reported to show frequent mutations in melanoma [32]. The expression of these genes was associated with vascular function, and the development of hypoxia in melanoma xenografts. However further studies are needed to determine whether there is a causal relationship between these parameters.

We have previously shown that angiogenesis can be inhibited by targeting the VEGFA pathway in A-07 tumors, and this treatment reduced vessel densities and induced hypoxia [33]. We have also demonstrated that artificially imposed hypoxia (i.e., by exposing tumorbearing mice to a low oxygen atmosphere) can increase VEGFA expression and induce angiogenesis in the same tumor model [34]. These experiments illustrate the complex interplay between angiogenesis, vasculature, and hypoxia in tumors, and imply that several experiments with different experimental design may be needed to determine causal relationships.

Clinical investigations have revealed that low oxygen tension and hypoxic tumor regions are characteristic features of the physiological microenvironment of human melanomas $[35,36]$. Various antiangiogenic drugs targeting the VEGFA pathway have been evaluated in clinical trials but none of the drugs have improved survival for patients with malignant melanoma [25]. The current study identifies other angiogenic stimulators than VEGFA that are associated with vascular function and the development of hypoxia in microscopic melanoma xenografts. It is possible that antiangiogenic drugs targeting these angiogenic pathways can be effective in human melanomas, and that such drugs can improve vascular function and tumor oxygenation. In line with this speculation, it has been demonstrated that low doses of antiangiogenic agents can improve vascular function and increase oxygenation in several experimental tumor models, including human ovarian carcinoma xenografts, human neuroblastoma xenografts, human glioma xenografts, murine melanoma, and murine breast carcinoma [37-39].

\section{Conclusions}

Two of the four investigated melanoma xenograft models developed hypoxic regions before they reached a size of $2-3 \mathrm{~mm}$ in diameter. Poor vascular pericyte coverage and inadequate vascular function were associated with the development of hypoxia in microscopic tumors, and the vascular abnormalities were associated with the angiogenic profile of the melanoma xenograft models.

\section{Abbreviations}

bFGF: basic fibroblast growth factor; BST: blood supply time; EREG: epiregulin; HAND2: basic helix-loop-helix transcription factor; $\mathrm{HF}_{\mathrm{PIM}}$ : fraction of pimonidazole-positive cells; $\mathrm{HF}_{\mathrm{RAD}}$ : fraction of radiobiologically hypoxic cells; NRP1: neuropilin 1; TGFBR1: transforming growth factor $\beta$ receptor 1 ; uPA: urokinasetype plasminogen activator; VEGF: vascular endothelial growth factor.

\section{Authors' contributions}

JVG, TGS, and EKR conceived and designed the study. JVG and LMKA performed the experiments. JVG, TGS, and EKR analyzed and interpreted the data. JVG wrote the manuscript. All authors read and approved the final manuscript.

\section{Acknowledgements}

Not applicable.

\section{Competing interests}

The authors declare that they have no competing interests.

\section{Availability of data and materials}

The datasets generated during and/or analysed during the current study are available from the corresponding author on reasonable request.

\section{Consent for publication}

Not applicable.

\section{Ethics approval and consent to participate}

The animal experiments were approved by the Norwegian National Animal Research Authority and were done according to the Interdisciplinary Principles and Guidelines for the Use of Animals in Research, Marketing, and Education (New York Academy of Sciences, New York, NY)

\section{Funding}

Financial support was received from the Norwegian Cancer Society and the South-Eastern Norway Regional Health Authority.

\section{Publisher's Note}

Springer Nature remains neutral with regard to jurisdictional claims in published maps and institutional affiliations.

Received: 11 August 2017 Accepted: 21 November 2017

Published online: 28 November 2017 


\section{References}

1. Vaupel P, Mayer A. Hypoxia in cancer: significance and impact on clinical outcome. Cancer Metastasis Rev. 2007:26:225-39.

2. Brown JM, Giaccia AJ. The unique physiology of solid tumors: opportunities (and problems) for cancer therapy. Cancer Res. 1998;58:1408-16.

3. Facciabene A, Peng X, Hagemann IS, Balint K, Barchetti A, Wang LP, Gimotty PA, Gilks CB, Lal P, Zhang L, Coukos G. Tumour hypoxia promotes tolerance and angiogenesis via CCL28 and Treg cells. Nature. 2011:475:226-30.

4. Rofstad EK. Microenvironment-induced cancer metastasis. Int J Radiat Biol. 2000:76:589-605

5. Vaupel P, Kallinowski F, Okunieff P. Blood flow, oxygen and nutrient supply, and metabolic microenvironment of human tumors: a review. Cancer Res. 1989;49:6449-65.

6. Dewhirst MW, Ong ET, Braun RD, Smith B, Klitzman B, Evans SM, Wilson D. Quantification of longitudinal tissue $\mathrm{pO} 2$ gradients in window chamber tumours: impact on tumour hypoxia. Br J Cancer. 1999;79:1717-22.

7. Jain RK. Determinants of tumor blood flow: a review. Cancer Res. 1988;48:2641-58.

8. Moulder JE, Rockwell S. Hypoxic fractions of solid tumors: experimentaltechniques, methods of analysis, and a survey of existing data. Int J Radiat Oncol Biol Phys. 1984;10:695-712.

9. Li XF, Carlin S, Urano M, Russell J, Ling CC, O'donoghue JA. Visualization of hypoxia in microscopic tumors by immunofluorescent microscopy. Cancer Res. 2007;67:7646-53.

10. Rofstad EK. Orthotopic human melanoma xenograft model systems for studies of tumour angiogenesis, pathophysiology, treatment sensitivity and metastatic pattern. Br J Cancer. 1994;70:804-12.

11. Gaustad JV, Brurberg KG, Simonsen TG, Mollatt CS, Rofstad EK. Tumor vascularity assessed by magnetic resonance imaging and intravital microscopy imaging. Neoplasia. 2008;10:354-62.

12. Øye KS, Gulati G, Graff BA, Gaustad JV, Brurberg KG, Rofstad EK. A novel method for mapping the heterogeneity in blood supply to normal and malignant tissues in the mouse dorsal window chamber. Microvasc Res. 2008;75:179-87.

13. Rofstad EK, Måseide K. Radiobiological and immunohistochemical assessment of hypoxia in human melanoma xenografts: acute and chronic hypoxia in individual tumours. Int J Radiat Biol. 1999;75:1377-93.

14. Simonsen TG, Gaustad JV, Leinaas MN, Rofstad EK. High interstitial fluid pressure is associated with tumor-line specific vascular abnormalities in human melanoma xenografts. PLoS ONE. 2012;7:e40006.

15. Fenton BM, Kiani MF, Siemann DW. Should direct measurements of tumor oxygenation relate to the radiobiological hypoxic fraction of a tumor. Int $J$ Radiat Oncol Biol Phys. 1995:33:365-73.

16. Rofstad EK, Mathiesen B, Galappathi K. Increased metastatic dissemination in human melanoma xenografts after subcurative radiation treatment: radiation-induced increase in fraction of hypoxic cells and hypoxia-induced up-regulation of urokinase-type plasminogen activator receptor. Cancer Res. 2004;64:13-8.

17. Gaustad JV, Simonsen TG, Andersen LMK, Rofstad EK. The effect of sunitinib treatment in human melanoma xenografts: associations with angiogenic profiles. Transl Oncol. 2017;10:158-67.

18. Tozer GM, Ameer-Beg SM, Baker J, Barber PR, Hill SA, Hodgkiss RJ, Locke $R$, Prise VE, Wilson I, Vojnovic B. Intravital imaging of tumour vascula networks using multi-photon fluorescence microscopy. Adv Drug Deliv Rev. 2005;57:135-52.

19. Groebe K, Vaupel P. Evaluation of oxygen diffusion distances in humanbreast cancer xenografts using tumor-specific invivo data: role of various mechanisms in the development of tumor hypoxia. Int J Radiat Oncol Biol Phys. 1988;15:691-7.

20. Armulik A, Genove G, Betsholtz C. Pericytes: developmental, physiological, and pathological perspectives, problems, and promises. Dev Cell. 2011;21:193-215
21. Keskin D, Kim J, Cooke VG, Wu CC, Sugimoto H, Gu CH, De Palma M, Kalluri R, LeBleu VS. Targeting vascular pericytes in hypoxic tumors increases lung metastasis via angiopoietin-2. Cell Rep. 2015;10:1066-81.

22. Cooke VG, LeBleu VS, Keskin D, Khan Z, O'Conne JT, Teng Y, Duncan MB, Xie L, Maeda G, Vong S, et al. Pericyte depletion results in hypoxia-associated epithelial-to-mesenchymal transition and metastasis mediated by met signaling pathway. Cancer Cell. 2012;21:66-81.

23. Cao YT, Li CY, Moeller BJ, Yu DH, Zhao YL, Dreher MR, Shan SQ, Dewhirst MW. Observation of incipient tumor angiogenesis that is independent of hypoxia and hypoxia inducible factor-1 activation. Cancer Res. 2005;65:5498-505.

24. Kaanders JHAM, Bussink J, van der Kogel AJ. Clinical studies of hypoxia modification in radiotherapy. Semin Radiat Oncol. 2004;14:233-40.

25. Jour G, Ivan D, Aung PP. Angiogenesis in melanoma: an update with a focus on current targeted therapies. J Clin Pathol. 2016;69:472-83.

26. Riese DJ, Cullum RL. Epiregulin: roles in normal physiology and cancer. Semin Cell Dev Biol. 2014;28:49-56.

27. Yamagishi H, Olson EN, Srivastava D. The basic helix-loop-helix transcription factor, dHAND, is required for vascular development. J Clin Investig. 2000;105:261-70.

28. Lampropoulou A, Ruhrberg C. Neuropilin regulation of angiogenesis. Biochem Soc Trans. 2014:42:1623-8.

29. Su SC, Lin CW, Yang WE, Fan WL, Yang SF. The urokinase-type plasminogen activator (UPA) system as a biomarker and therapeutic target in human malignancies. Expert Opin Ther Targets. 2016;20:551-66.

30. Lohela M, Bry M, Tammela T, Alitalo K. VEGFs and receptors involved in angiogenesis versus lymphangiogenesis. Curr Opin Cell Biol. 2009;21:154-65.

31. Khan Z, Marshall JF. The role of integrins in TGF beta activation in the tumour stroma. Cell Tissue Res. 2016;365:657-73.

32. Hayward NK, Wilmott JS, Waddell N, Johansson PA, Field MA, Nones K, Patch AM, Kakavand H, Alexandrov LB, Burke H, et al. Whole-genome landscapes of major melanoma subtypes. Nature. 2017:545:175-80.

33. Gaustad JV, Pozdniakova V, Hompland T, Simonsen TG, Rofstad EK. Magnetic resonance imaging identifies early effects of sunitinib treatment in human melanoma xenografts. J Exp Clin Cancer Res. 2013;32:93.

34. Rofstad EK, Gaustad JV, Egeland TA, Mathiesen B, Galappathi K. Tumors exposed to acute cyclic hypoxic stress show enhanced angiogenesis, perfusion and metastatic dissemination. Int J Cancer. 2010;127:1535-46.

35. Hartmann P, Mirtolouei R, Untersberger S, Ziegler W, Hermann Z, Richtig E, Hofmann-Wellenhof R, Grinschgl S, Kerl H, Smolle J. Non-invasive imaging of tissue PO2 in malignant melanoma of the skin. Melanoma Res. 2006;16:479-86

36. Lartigau E, Randrianarivelo H, Avril MF, Margulis A, Spatz A, Eschwege F, Guichard M. Intratumoral oxygen tension in metastatic melanoma. Melanoma Res. 1997:7:400-6.

37. Dings RP, Loren M, Heun H, McNiel E, Griffioen AW, Mayo KH, Griffin RJ. Scheduling of radiation with angiogenesis inhibitors anginex and Avastin improves therapeutic outcome via vessel normalization. Clin Cancer Res. 2007;13:3395-402.

38. Dickson PV, Hamner JB, Sims TL, Fraga CH, Ng CY, Rajasekeran S, Hagedorn NL, McCarville MB, Stewart CF, Davidoff AM. Bevacizumab-induced transient remodeling of the vasculature in neuroblastoma xenografts results in improved delivery and efficacy of systemically administered chemotherapy. Clin Cancer Res. 2007:13:3942-50.

39. Winkler F, Kozin SV, Tong RT, Chae SS, Booth MF, Garkavtsev I, Xu L, Hicklin DJ, Fukumura D, di Tomaso E, et al. Kinetics of vascular normalization by VEGFR2 blockade governs brain tumor response to radiation: role of oxygenation, angiopoietin-1, and matrix metalloproteinases. Cancer Cell. 2004;6:553-63. 